\title{
A Spiral Spin-Echo MR Imaging Technique for Improved Flow Artifact Suppression in T1-Weighted Postcontrast Brain Imaging: A Comparison with Cartesian Turbo Spin-Echo
}

\author{
(D) Z. Li, (D)H.H. Hu, (D).H. Miller, (D).P. Karis, DP. Cornejo, (DD. Wang, and (DJ.G. Pipe
}

\begin{abstract}
BACKGROUND AND PURPOSE: A challenge with the T1-weighted postcontrast Cartesian spin-echo and turbo spin-echo brain MR imaging is the presence of flow artifacts. Our aim was to develop a rapid 2D spiral spin-echo sequence for Tl-weighted MR imaging with minimal flow artifacts and to compare it with a conventional Cartesian 2D turbo spin-echo sequence.
\end{abstract}

MATERIALS AND METHODS: T1-weighted brain imaging was performed in 24 pediatric patients. After the administration of intravenous gadolinium contrast agent, a reference Cartesian TSE sequence with a scanning time of 2 minutes 30 seconds was performed, followed by the proposed spiral spin-echo sequence with a scanning time of 1 minutes 18 seconds, with similar spatial resolution and volumetric coverage. The results were reviewed independently and blindly by 3 neuroradiologists. Scores from a 3-point scale were assigned in 3 categories: flow artifact reduction, subjective preference, and lesion conspicuity, if any. The Wilcoxon signed rank test was performed to evaluate the reviewer scores. The $t$ test was used to evaluate the SNR. The Fleiss $\kappa$ coefficient was calculated to examine interreader agreement.

RESULTS: In 23 cases, spiral spin-echo was scored over Cartesian TSE in flow artifact reduction $(P<.001)$. In 21 cases, spiral spin-echo was rated superior in subjective preference $(P<.001)$. Ten patients were identified with lesions, and no statistically significant difference in lesion conspicuity was observed between the 2 sequences. There was no statistically significant difference in SNR between the 2 techniques. The Fleiss $\kappa$ coefficient was 0.79 ( $95 \%$ confidence interval, 0.65-0.93).

CONCLUSIONS: The proposed spiral spin-echo pulse sequence provides postcontrast images with minimal flow artifacts at a faster scanning time than its Cartesian TSE counterpart.

ABBREVIATION: $\mathrm{SE}=$ spin-echo

$\mathrm{T}$ 1-weighted MR imaging after the injection of gadoliniumbased contrast agent is widely used in the diagnosis of many neurologic diseases, such as tumors, infections, and inflammatory conditions. 2D multisection Cartesian spin-echo (SE) and turbo spin-echo-based pulse sequences are the clinically preferred methods for postcontrast T1WI. A challenge with these Cartesian

Received May 28, 2015; accepted after revision August 19.

From the Departments of Imaging Research (Z.L., D.W., J.G.P.) and Radiology (J.P.K.), Barrow Neurological Institute, Phoenix, Arizona; and Department of Radiology (H.H.H., J.H.M., P.C.), Phoenix Children's Hospital, Phoenix, Arizona.

Z. Li and H.H. Hu are co-first authors.

Z.L., H.H.H., J.H.M, D.W., and J.G.P. receive research funding support from Philips Healthcare.

Paper previously presented as electronic poster No. 135 at: American Society of Neuroradiology Annual Meeting and the Foundation of the ASNR Symposium, April 25-30, 2015; Chicago, Illinois.

Please address correspondence to Zhiqiang Li, PhD, Barrow Neurological Institute, Keller Center for Imaging Innovation, 350 W Thomas Rd, Phoenix AZ 85013; e-mail: lizhiqiang@gmail.com

http://dx.doi.org/10.3174/ajnr.A4600 images is the presence of ghosting artifacts due to flowing blood from the venous sinuses. These artifacts can obscure the visualization of lesions and reduce image quality. With contrast-agent enhancement, these flow artifacts are further exacerbated by bright-blood signals. Gradient flow compensation and spatial saturation bands are helpful in alleviating, but not eliminating, these flow-induced artifacts in Cartesian acquisitions.

Spiral MR imaging, a non-Cartesian acquisition technique, has several advantages over its Cartesian counterpart. ${ }^{1,2}$ A primary benefit is the ability of the spiral to traverse $k$-space more efficiently per unit of time than Cartesian trajectories, thus providing a higher scan speed. With spiral acquisitions, motion- and flow-induced errors are manifest as incoherent artifacts in the image domain. As a result, spiral acquisition reduces the sensitivity of the pulse sequence to structured artifacts. ${ }^{3}$ The spiral trajectory also inherently provides zero gradient moments at the origin of $k$-space, which substantially decreases the sensitivity of the sequence to in-plane flow-related artifacts. ${ }^{4}$ Spiral SE MR imaging 


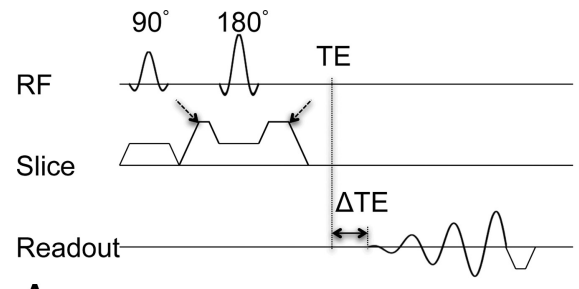

A spiral SE sequence

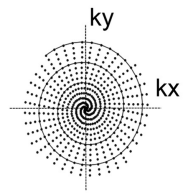

B spiral k-space

FIG 1. Pulse sequence diagram of the proposed 2D spiral SE sequence (A). $\Delta T E$ indicates the temporal shift of the spiral readout relative to the traditional TE (ie, the Hahn echo). Spiral data at multiple $\Delta$ TE shifts $(-0.2,0.57$, and $1.34 \mathrm{~ms}$ used at $3 \mathrm{~T})$ were collected in this work to facilitate chemical shift-encoded water-fat imaging. The dashed arrows point to the crusher gradients in the section direction used to spoil through-plane flow signal. B, The solid circular tracing shows 1 spiral interleaf, and the dots indicate sampled data points in $k$-space from all interleaves, as summarized in Table 1. RF indicates radiofrequency.

Table 1: 3T imaging parameters of conventional 2D multisection Cartesian TSE and the proposed 2D spiral SE

\begin{tabular}{lcc}
\multicolumn{1}{c}{ Parameter } & Cartesian TSE & Spiral SE \\
\hline Orientation & Axial & Axial \\
Phase-encoding direction & Anterior/posterior & NA \\
FOV $(\mathrm{mm})$ & $220 \times 220$ & $220 \times 220$ \\
In-plane resolution $(\mathrm{mm})$ & $0.8 \times 1.0$ & $0.85 \times 0.85$ \\
Section thickness $(\mathrm{mm})$ & 3 & 3 \\
Section gap $(\mathrm{mm})$ & 0.3 & 0.3 \\
No. of sections & 15 & 15 \\
Excitation flip angle & $70^{\circ}$ & $90^{\circ}$ \\
TE (ms) & 9 & 10 \\
$\Delta$ TE (ms) & $0,1.0$ & $-0.2,0.57,1.34$ \\
Echo-train length & 3 & $\mathrm{NA}$ \\
TR (ms) & 674 & 674 \\
No. of spiral interleaves & $\mathrm{NA}$ & 38 \\
Gradient flow & On & Off \\
compensation & & \\
Spatial saturation band & On & Off \\
No. of section packages & 2 & 1 \\
Scanning time & 2 min $30 \mathrm{sec}$ & min $18 \mathrm{sec}$ \\
\hline
\end{tabular}

Note:-NA indicates not applicable; $\Delta$ TE, TE shifts.

${ }^{a}$ Note the use of gradient flow compensation and a spatial saturation band with the Cartesian TSE technique, whereas they are absent in the spiral SE sequence. Note also differences in scanning times despite comparable spatial resolution and section coverage.

has been reported in pelvic imaging, ${ }^{5}$ black-blood imaging of peripheral vasculature, ${ }^{6}$ and functional MR imaging. ${ }^{7}$

The purpose of this work was to develop a 2D spiral SE technique for T1-weighted brain imaging with minimal flow artifacts and faster scanning speed and compare it with a conventional 2D Cartesian TSE pulse sequence, with comparable spatial resolution and volumetric coverage. We prospectively evaluated the performance of the 2D spiral SE technique and its subsequent image quality in a cohort of pediatric patients.

\section{MATERIALS AND METHODS}

This prospective, Health Insurance Portability and Accountability Act-compliant study was approved by the Phoenix Children's Hospital and the Barrow Neurological Institute review boards under expedited review as a minimum-risk study, and informed consent was waived from patients at the Phoenix Children's Hospital for adding the proposed spiral sequence to a standard brain MR imaging examination.

\section{D Spiral SE MR Imaging}

To overcome slower scan speeds and pronounced flow artifacts in conventional Cartesian TSE and SE techniques in postcontrast T1WI, we proposed, in this work, a SE pulse sequence by using a spiral-out readout, as shown in Fig 1. In Cartesian imaging, motion- and flow-induced errors express themselves as coherent ghosting artifacts along the phase-encoding direction. In spiral imaging, there is no defined phase-encoding direction (Fig 1B). Therefore, motion- and flow-induced errors are manifest in all directions as incoherent artifacts. The reduced sensitivity to inplane flow-induced artifacts is also because the gradient moments at the beginning of the spiral readout are nulled for all orders (ie, static, velocity, acceleration, jerk, and so forth), which leads to reduced phase errors at the center of $k$-space. Thus, the proposed spiral SE sequence obviates additional gradient flow compensation, typically used in Cartesian TSE to suppress in-plane flow signals. An additional measure used in this work was large crusher gradients along the section direction around the $180^{\circ}$ refocusing radiofrequency pulse of the SE. These further reduce throughplane flow-induced artifacts. ${ }^{6}$ The overall reduced sensitivity of spiral SE to flow-induced errors eliminates the need for additional spatial saturation bands that are typically used in Cartesian TSE acquisitions to null signals from through-plane inflowing blood. Removal of these saturation bands in spiral SE also reduces magnetization transfer effects and improves the gray-white matter tissue contrast.

\section{Water-Fat Imaging Capability}

In this work, multiecho chemical shift-encoded Dixon water-fat imaging capability ${ }^{8,9}$ was added to the proposed spiral SE technique; thus, it is similar to the conventional Cartesian TSE sequence of our institution, the comparison reference, which has built-in Dixon capability from the manufacturer. Three echoes were used in the spiral SE pulse sequence to achieve reliable waterfat separation and deblurring. ${ }^{10}$

\section{Data Acquisition}

All data were acquired on two 3T scanners (Ingenia; Philips Healthcare, Best, the Netherlands) by using either a 13- or a 32-channel head array. In each patient, the axial 2D Cartesian TSE scan was obtained first after intravenous contrast (gadopentetate dimeglumine, Magnevist; Bayer HealthCare Pharmaceuticals, Wayne, New Jersey) administration, followed by the proposed spiral SE scan. The imaging parameters are summarized in Table 1.

\section{SNR Evaluation}

To compare the SNR of the spiral SE and Cartesian TSE sequences, we initially scanned 2 healthy adult volunteers, without the use of intravenous contrast. SNR can typically be measured by estimating the noise SD from either the background region or the difference of 2 consecutive image sets acquired under identical conditions. ${ }^{11,12}$ Because the background air regions in spiral reconstructed images do not contain pure Gaussian noise, the latter method of acquiring 2 identical image sets was adopted in this work, and the difference of the 2 images was used to estimate noise and ultimately SNR. In each volunteer, areas with relatively uni- 


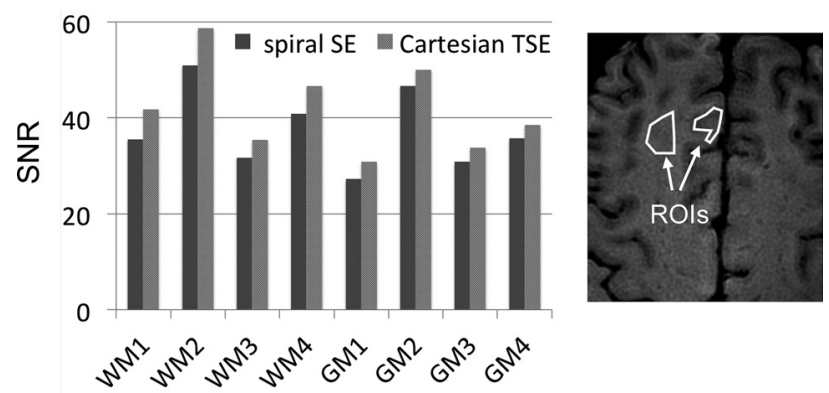

FIG 2. Measured SNR in 8 ROls ( 4 white matter and 4 gray matter). The ROI size range was $269-1080$ voxels with a mean of 530 voxels (range, 56.5-226.9 mm², with mean of $111.3 \mathrm{~mm}^{2}$ ), with 1 example shown on the right. The relative SNR of spiral SE to Cartesian TSE was $89.3 \%$ $\pm 3.0 \%$.

form gray or white matter signals were selected from the spiral SE image, and ROIs were manually drawn. Selected areas included cortical gray matter, subcortical white matter, deep white matter (centrum semiovale), and so forth, and 1 example was illustrated in Fig 2. The ROIs and signal and noise measurements were made by using Image J software (National Institutes of Health, Bethesda, Maryland).

\section{Patient Studies}

Twenty-four pediatric patients (18 boys, 6 girls) were prospectively enrolled in this study from October to December 2014. There were no specific inclusion/exclusion criteria. The age ranged from 7 months to 13 years 8 months, with a distribution of $6.1 \pm 3.9$ years. Twenty-two of the 24 patients were scanned with sedation. The cohort is briefly summarized in Table 2 .

\section{Data Analysis}

The water-reconstructed images from Cartesian TSE and spiral SE were independently assessed by 3 radiologists, with 2 (P.C.), 9 (J.H.M.), and 23 (J.P.K.) years of experience in neuroradiology, respectively. The images were rated in 3 categories, including flow artifact reduction, subjective preference (meaning the radiologists would rather interpret one image than the other), and lesion conspicuity, if present. For each patient, the readers were given the combined dataset (Cartesian TSE and spiral SE) in a 2-column format with the left-right order randomized as either Cartesian TSE/spiral SE or spiral SE/Cartesian TSE. The readers reviewed all images (ie, all sections), and an overall score was given for each patient in each category by using a 3-point score system, with -1 representing the left superior to the right, zero representing the left equivalent to the right, and 1 representing the left inferior to the right. The scores were then converted by the remaining investigators in a separate session so that -1 denoted Cartesian TSE superior to spiral SE, zero denoted Cartesian TSE equivalent to spiral SE, and 1 denoted Cartesian TSE inferior to spiral SE.

\section{Statistical Analysis}

The SPSS software package (Version 15.0; IBM, Armonk, New York) was used for statistical analysis. The $t$ test was used to assess any statistically significant difference in SNR between Cartesian TSE and spiral SE. We used the nonparametric 1-sample Wilcoxon signed rank test to determine whether the scores from each of the 3 radiologists for each of the 3 categories differed from zero,
Table 2: Summary of study cohort

\begin{tabular}{|c|c|c|c|}
\hline Patient & Sex & Age & $\begin{array}{l}\text { Clinical Indication for } \\
\text { Brain MRI Exam }\end{array}$ \\
\hline 1 & $M$ & $2 \mathrm{yr} 6 \mathrm{mo}$ & Optic nerve hypoplasia \\
\hline $2^{\mathrm{a}}$ & M & $1 \mathrm{yr} 7 \mathrm{mo}$ & $\begin{array}{l}\text { Edema, craniosynostosis repair, } \\
\text { head swelling, fever }\end{array}$ \\
\hline $3^{b}$ & M & $12 \mathrm{yr} 7 \mathrm{mo}$ & Hydrocephalus \\
\hline 4 & M & 10 yr 0 mo & Suspicion of brain tumor \\
\hline 5 & M & 3 yr 2 mo & $\begin{array}{l}\text { Hemiparesis dominant left } \\
\text { side }\end{array}$ \\
\hline $6^{\mathrm{a}, \mathrm{b}}$ & $\mathrm{F}$ & $9 \mathrm{yr} 4 \mathrm{mo}$ & Spell convulsion \\
\hline 7 & M & 13 yr 8 mo & Suspicion of brain tumor \\
\hline $8^{a}$ & $\mathrm{~F}$ & 5 yr 5 mo & Hearing loss \\
\hline 9 & M & $0 \mathrm{yr} 10 \mathrm{mo}$ & Adrenogenital disorders \\
\hline $10^{a}$ & M & $3 \mathrm{yr} 2 \mathrm{mo}$ & Bilateral retinoblastoma \\
\hline 11 & M & 4 yr 0 mo & Cerebellar ataxia \\
\hline 12 & M & $0 \mathrm{yr} 7 \mathrm{mo}$ & Adduction deficit \\
\hline 13 & $\mathrm{~F}$ & $8 \mathrm{yr}$ & Hearing loss \\
\hline 14 & M & $8 \mathrm{yr} 7 \mathrm{mo}$ & Papilledema \\
\hline $15^{\mathrm{a}}$ & M & $7 \mathrm{yr} 8 \mathrm{mo}$ & Low-grade glioma \\
\hline 16 & M & $5 \mathrm{yr} 2 \mathrm{mo}$ & $\begin{array}{l}\text { Central nervous system } \\
\text { tumor mass }\end{array}$ \\
\hline 17 & $\mathrm{~F}$ & $2 \mathrm{yr} 6 \mathrm{mo}$ & Fever, meningitis \\
\hline $18^{\mathrm{a}}$ & $\mathrm{F}$ & $2 \mathrm{yr} 1 \mathrm{mo}$ & Epilepsy \\
\hline 19 & $\mathrm{~F}$ & $4 \mathrm{yr} 3 \mathrm{mo}$ & Headache \\
\hline $20^{\mathrm{a}}$ & M & $11 \mathrm{yr} 2 \mathrm{mo}$ & $\begin{array}{l}\text { Ablepharon-macrostomia } \\
\text { syndrome, encephalopathy }\end{array}$ \\
\hline $21^{\mathrm{a}}$ & M & $9 \mathrm{yr} 1 \mathrm{mo}$ & Malignant neoplasm of brain \\
\hline 22 & M & $11 \mathrm{yr} 3 \mathrm{mo}$ & Headache \\
\hline $23^{a}$ & M & $3 \mathrm{yr} 1 \mathrm{mo}$ & Brain tumor, infection \\
\hline $24^{\mathrm{a}}$ & M & $6 \mathrm{yr} 7 \mathrm{mo}$ & Intracranial abscess, mastoiditis \\
\hline
\end{tabular}

a Patients with space-occupying lesions or signal abnormalities seen on MRI.

${ }^{b}$ Patients who were not sedated.

where zero implied similarity between the spiral SE and Cartesian TSE images. A $P$ value $<.05$ was chosen to reflect statistical significance. Interradiologist agreement was evaluated with the Fleiss $\kappa$ coefficient.

\section{RESULTS}

Tables 1 and 2 summarize pertinent imaging parameters and descriptors of the study cohort, respectively. Figure 2 illustrates SNR measurements in the gray and white matter of healthy volunteers between spiral SE and Cartesian TSE sequences. The $t$ test did not yield a statistically significant difference in SNR between spiral SE and Cartesian TSE $(P=0.32)$ when considering all gray and white matter ROIs. However, the SNR of the spiral SE approach was slightly lower, and the SNR of the spiral SE to Cartesian TSE was $89.3 \% \pm 3.0 \%$. The main reason for the lower SNR with the spiral SE acquisition is its shorter scanning time (Table 1).

Scores from radiologists' evaluations are outlined in Fig 3. While the categories of flow artifact reduction and subjective preference included all 24 patient datasets, the category of lesion conspicuity was limited to 10 cases because only these cases were identified as having either contrast-enhancing ( 6 cases) or noncontrast-enhancing ( 4 cases) pathology, none of which was in the posterior fossa. All 3 observers rated spiral SE better than Cartesian TSE in terms of flow artifacts in 23 of the 24 cases (Fig $3 A$ ). In the remaining case, 2 readers rated both techniques as equivalent in flow artifact reduction, while the third reader preferred the proposed spiral SE. The improvement in flow artifact reduction with spiral SE over Cartesian TSE was statistically significant $(P<$ $.001)$. 


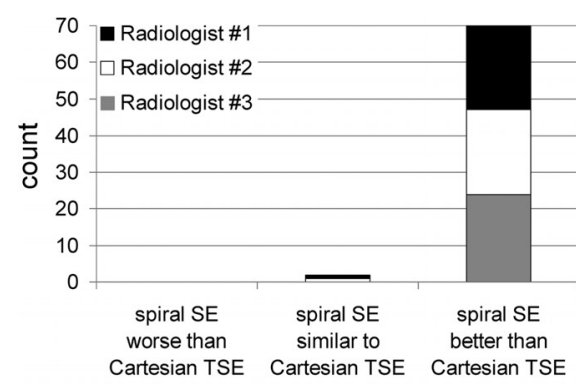

A

Flow Artifact Reduction

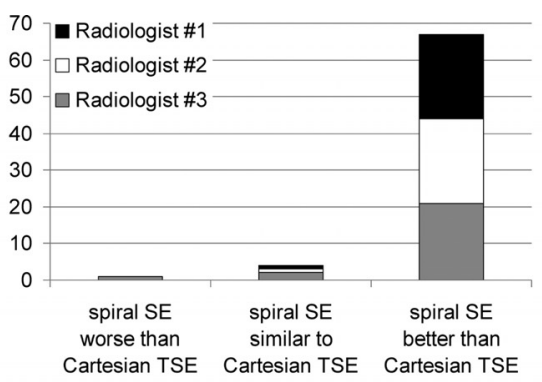

B

\section{Subjective Preference}

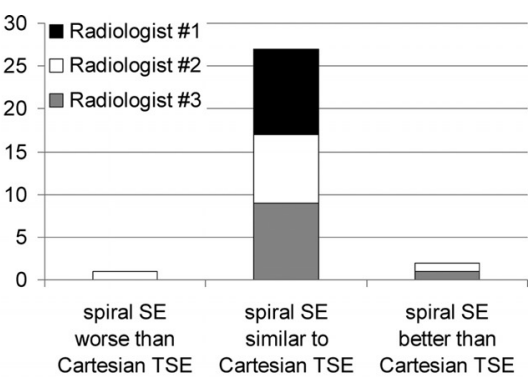

C Lesion Conspicuity

FIG 3. Barplots summarizing the score assessment of patient data by 3 radiologists, in terms of flow artifact reduction ( $A$ ), subjective preference $(B)$, and lesion conspicuity $(C)$. In the categories of flow artifact reduction $(A)$ and subjective preference $(B)$, the maximum count per radiologist is 24,1 for each patient. Thus, the cumulative count is 72 . In the category of lesion conspicuity $(C)$, which was evaluated in a subgroup of 10 patients, the maximum count of each radiologist is 10 , with a cumulative total of 30 . Note the preference of the spiral SE images in $A$ and $B$.

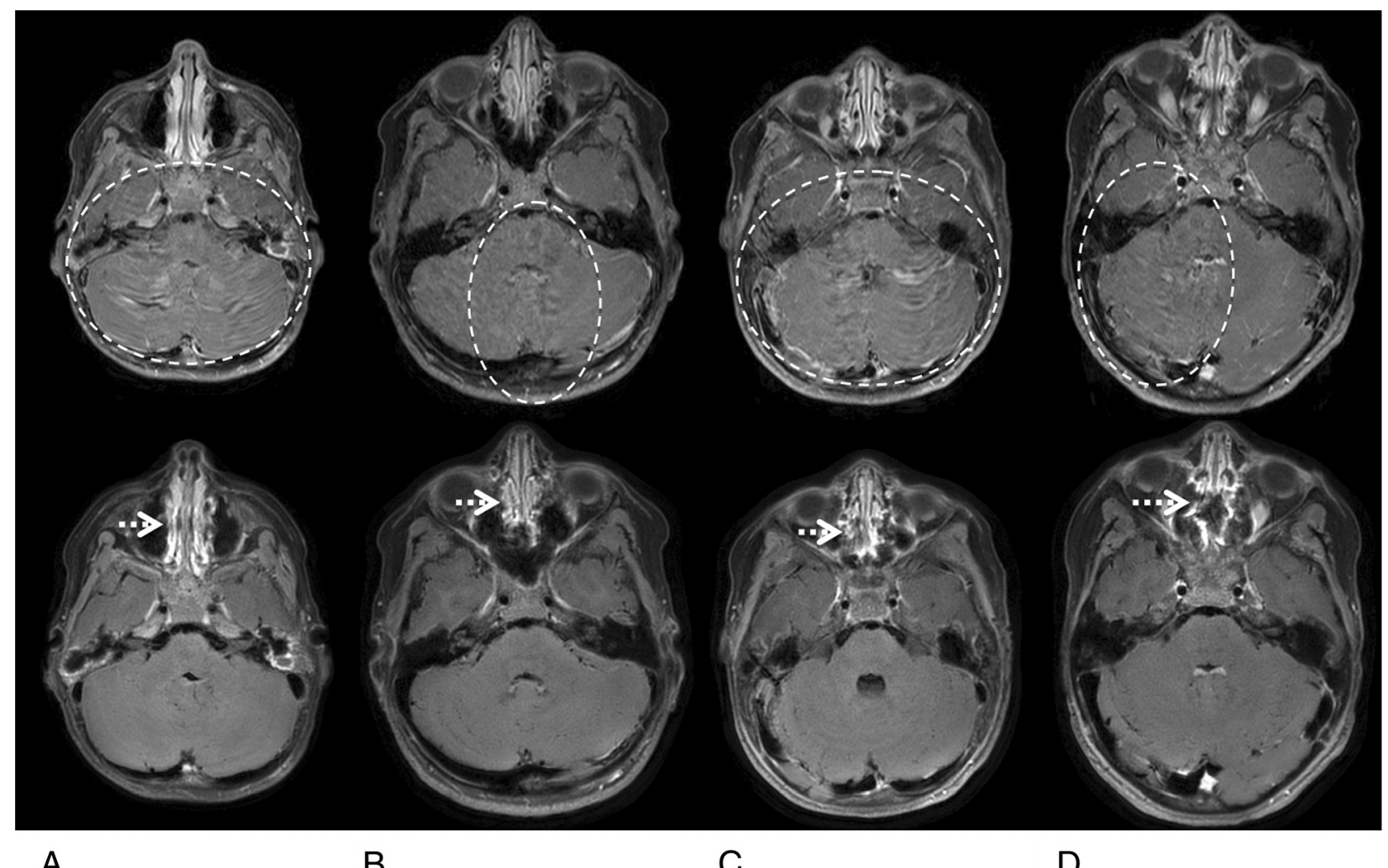

A

B

C

FIG 4. Representative water-reconstructed images from patients $1(A), 4(B), 18(C)$, and $22(D)$, comparing flow artifacts between Cartesian TSE (top) and spiral SE sections (bottom). In the Cartesian TSE images, the dashed circles denote areas with noticeable flow artifacts, seen predominantly as signal ghosting. It is evident that the flow artifacts are substantially reduced in the corresponding spiral SE images, especially in the cerebellum where strong flow artifacts are typically observed in Cartesian TSE. The dotted arrows in the spiral SE images point to residual blurring in the nasal cavity, in comparison with the sharper appearance seen in the Cartesian TSE data.

In 21 of the 24 cases, the 3 radiologists preferred spiral SE over Cartesian TSE in terms of subjective preference (Fig 3B). In 2 cases, 2 reviewers preferred spiral SE, while the third reviewer rated both techniques equivalent. In another case, one reviewer preferred Cartesian TSE, while the other 2 reviewers rated both techniques equivalent. A statistically significant improvement with spiral SE over Cartesian TSE in subjective preference was observed $(P<.001)$.

In 8 of the 10 cases with lesions, spiral SE was rated by all 3 observers as equivalent to Cartesian TSE in providing lesion conspicuity (Fig 3C). In 1 case, 2 observers preferred spiral SE, while the third observer scored the 2 techniques as equivalent. In another case, 2 observers rated the 2 techniques equivalent while the third observer considered Cartesian TSE better. With this limited number of data points, the scores for lesion conspicuity between spiral SE and Cartesian TSE were not statistically different. The interreader Fleiss $\kappa$ coefficient was 0.79 ( $95 \%$ confidence interval, 0.65-0.93) for all 3 categories of flow artifact reduction, subjective preference, and lesion conspicuity.

Figure 4 illustrates representative examples, highlighting the suppression of flow artifacts with the proposed 2D spiral SE sequence. The Cartesian TSE images showed substantial flow arti- 


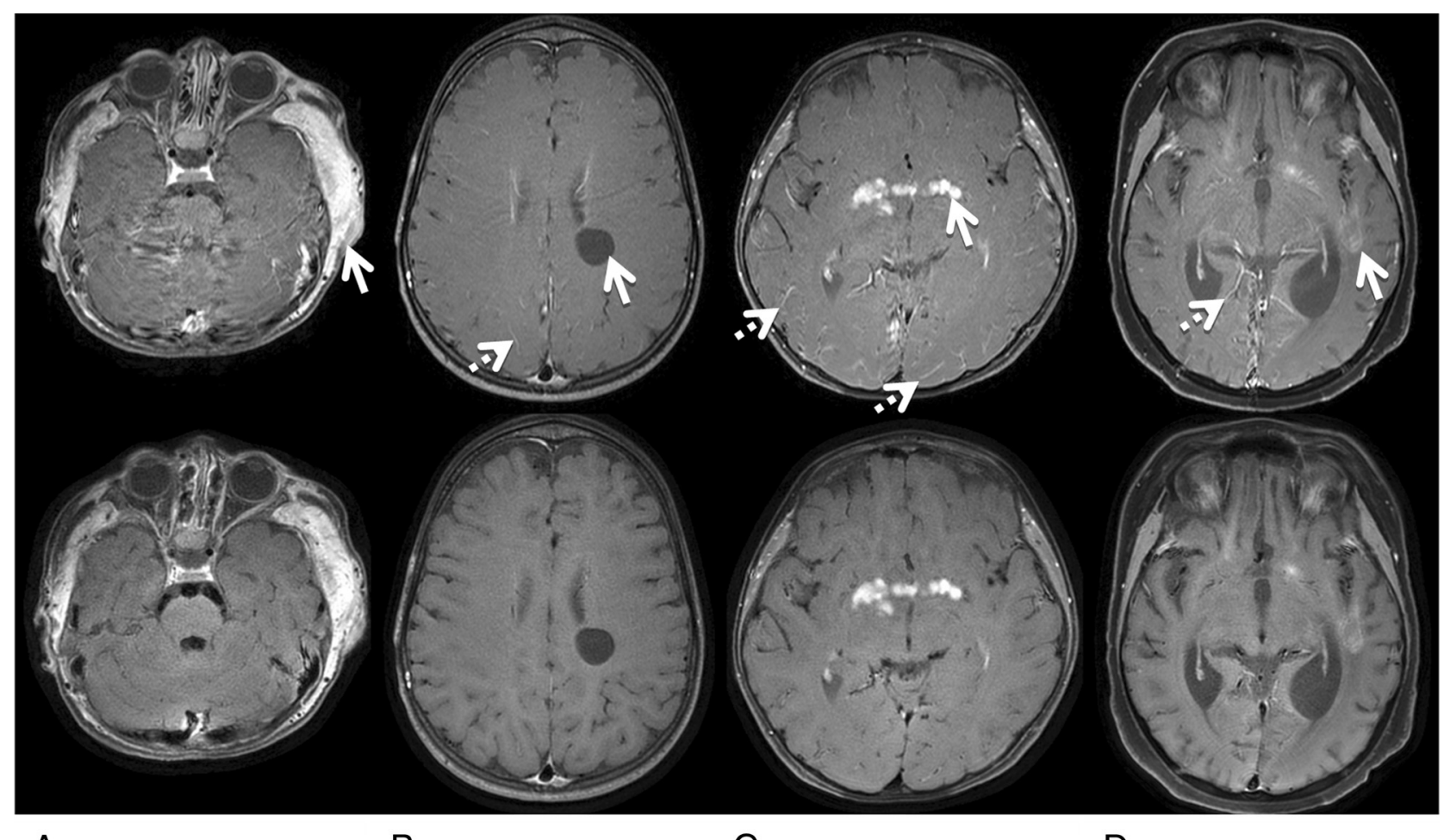

A

B

C

FIG 5. Representative images with lesions from patients $2(A), 8(B), 15(C)$, and $20(D)$, comparing lesion conspicuity between Cartesian TSE (top) and spiral SE sections (bottom). $A$, The MR image shows extracranial soft-tissue enhancement related to postsurgical infection. $B$, The patient has a nonenhancing neural cyst. $C$, The patient has an enhancing hypothalamic glioma. $D$, The patient has scattered enhancing intracranial leukemia tumors. The solid arrows in the Cartesian images point to these lesions, and the dotted arrows point to noticeable bright-blood signals in the small vessels that are absent in spiral SE images.

facts from the venous sinuses with resultant poor posterior fossa image quality. These artifacts were not present on the corresponding spiral SE images. Figure 5 shows 4 cases with intra- and extracranial contrast-enhancing and intracranial nonenhancing lesions. Lesion delineation is comparable between the spiral SE and Cartesian TSE data. Other observations include slightly higher gray-white matter tissue contrast and dark blood vessels in spiral SE images, in contrast to Cartesian TSE data.

\section{DISCUSSION}

Artifacts from pulsatile blood flow are a potential major concern in postcontrast T1WI Cartesian SE and TSE brain imaging. In this study, we have shown that a 2D spiral SE acquisition is capable of substantially reducing these flow artifacts, without compromising spatial resolution and volumetric coverage. The reduction in flow artifacts is achieved by exploiting the advantages of a spiral $k$ space readout. The spiral approach provides inherent gradient flow compensation and incoherent manifestation of residual artifacts. Through-plane flow signals were further reduced by the use of crusher gradients along the section-encoding axis. Parallel imaging was not used in this study but can be additionally applied to accelerate both techniques. In this work, spiral data were acquired with 3 TE shifts, which were necessary to achieve a robust water-fat separation. ${ }^{10}$ The algorithm ${ }^{10}$ with only 2 TE shifts can be used to further reduce scanning time. With this approach, however, an external B0 field map is required.

The SNR of the spiral SE technique was slightly lower than that of Cartesian TSE, albeit with a 2 -fold reduction in scanning time.
If SNR is more desirable than the scanning speed, it can be achieved by adjusting the image protocol. For instance, a gain of $\sqrt{ } 2$ in SNR can be obtained by acquiring data with a signal average of 2, which results in an SNR higher than the Cartesian TE, even though the total scanning time is comparable. Magnetization transfer effects are more pronounced in Cartesian $\mathrm{TSE}^{13}$ due to the use of multiple refocusing radiofrequency pulses and a spatial saturation band to spoil the inflow blood signal, which likely explains the gray-white matter contrast being slightly higher in the spiral SE data shown in Fig 5. The clinical utility of this magnetization transfer effect should be evaluated in the future.

The radiologists thought that the primary reason for the spiral images being preferred was due to the reduction in flow-related artifacts. Another factor was the black-blood vascular signal in the spiral data. Given the substantial reduction of flow artifacts and consistent black-blood signals with spiral SE data, it is plausible that any lesion obscured by flow artifacts or bright-blood signals in the Cartesian TSE acquisition can be more confidently detected with the spiral SE technique. Additional factors impacted the subjective preference, for instance, other forms of artifacts related to physiologic or bulk motion. These factors collectively contributed to the spiral SE acquisition being preferred over the Cartesian TSE approach by the evaluating radiologists. In current study, we did not adopt a more objective metric to compare the 2 techniques. Larger studies in the future should assess more objective and quantitative comparisons (eg, tissue contrast).

In this study, the 3 reviewing radiologists, though blinded and 
randomly presented with the data, may have been able to identify the spiral images on the basis of the significant reduction in flow artifacts. This potential source of bias in the evaluation might be difficult to avoid.

A limitation of this study was that no patients were found with lesions in the posterior fossa that was affected by strong flow artifacts in the Cartesian TSE datasets, and enhancing lesions were only found in a small number of cases. Although the study was limited to a cohort of pediatric patients, the present work is applicable to the general adult population. Future studies include the expansion to a large cohort of adult patients and include patients with brain parenchymal and high-flow vascular lesions. With a large patient population, seeing pathologies in the posterior fossa to prove the value of the improved flow artifact suppression with spiral SE is likely. Seeing more enhancing lesions is expected, making it feasible to statistically investigate the enhancement with spiral SE.

Another limitation was the fixed acquisition order (ie, Cartesian TSE followed by spiral SE after contrast administration). A randomized order would have permitted a more thorough analysis of the performance of the spiral SE sequence. In addition, the reference scan for comparison was a 2D Cartesian TSE acquisition, not a longer Cartesian SE pulse sequence. In the clinical setting of our institution, time did not permit performing the latter. In this study, spiral SE was not compared with gradientecho-based sequences, such as Cartesian MPRAGE. Performing such a comparison would be beneficial in future studies.

\section{CONCLUSIONS}

We have demonstrated a relatively simple 2D spiral SE approach in T1-weighted postcontrast brain MR imaging that has minimal flow artifacts in comparison with its 2D Cartesian TSE counterpart. 2D spiral SE can be performed more efficiently and provides faster scanning speed than 2D Cartesian TSE, without sacrificing spatial resolution or volumetric coverage.

\section{ACKNOWLEDGMENTS}

We thank Amber Pokorney at Phoenix Children's Hospital for assistance with patient data collection.
Disclosures: Zhiqiang Li-RELATED: Grant: As part of the group at the Barrow Neurological Institute, Z.L. received grant support from Philips Healthcare. * Houchun $\mathrm{H}$. Hu—RELATED: Grant: Philips Healthcare (research support).* Jeffrey H. Miller-RELATED: Consulting Fee or Honorarium: Philips Healthcare; Support for Travel to Meetings for the Study or Other Purposes: Philips Healthcare. Dinghui WangRELATED: Grant: research support from Philips Healthcare.* James G. Pipe-RELATED: Grant: Philips Healthcare. *Money paid to the institution.

\section{REFERENCES}

1. Ahn CB, Kim JH, Cho ZH. High-speed spiral-scan echo planar NMR imaging-I. IEEE Trans Med Imaging 1986;5:2-7 CrossRef Medline

2. Meyer $\mathrm{CH}, \mathrm{Hu} \mathrm{BS}$, Nishimura DG, et al. Fast spiral coronary artery imaging. Magn Reson Med 1992;28:202-13 CrossRef Medline

3. Bernstein MA, King KF, Zhou XJ. Handbook of MRI Pulse Sequence. San Diego: Elsevier Academic Press; 2004

4. Nishimura DG, Irarrazabal P, Meyer CH. A velocity k-space analysis of flow effects in echo-planar and spiral imaging. Magn Reson Med 1995;33:549-56 CrossRef Medline

5. Yacoe ME, Li KC, Cheung L, et al. Spiral spin-echo magnetic resonance imaging of the pelvis with spectrally and spatially selective radiofrequency excitation: comparison with fat-saturated fast spin-echo imaging. Can Assoc Radiol J 1997;48:247-51 Medline

6. Wang WT, Hu P, Meyer CH. Black-blood imaging using a spin-echo spiral sequence with flow-spoiling gradients. In: Proceedings of the Annual Meeting of International Society for Magnetic Resonance in Medicine, Seattle, Washington. May 6-12, 2006

7. Brewer KD, Rioux Ja, D‘Arcy CN, et al. Asymmetric spin-echo (ASE) spiral improves BOLD fMRI in inhomogeneous regions. NMR Biomed 2009;22:654-62 CrossRef Medline

8. Dixon WT. Simple proton spectroscopic imaging. Radiology 1984; 153:189-94 CrossRef Medline

9. Glover GH, Schneider E. Three-point Dixon technique for true water/fat decomposition with B0 inhomogeneity correction. Magn Reson Med 1991;18:371-83 CrossRef Medline

10. Wang D, Zwart NR, Li Z, et al. Analytical three-point Dixon method: with applications for spiral water-fat imaging. Magn Reson Med 2015 Mar 11. [Epub ahead of print] CrossRef Medline

11. Kaufman L, Kramer DM, Crooks LE, et al. Measuring signal-tonoise ratios in MR imaging. Radiology 1989;173:265-67 CrossRef Medline

12. Price RR, Axel L, Morgan T, et al. Quality assurance methods and phantoms for magnetic resonance imaging: report of AAPM nuclear magnetic resonance Task Group No. 1. Med Phys 1990;17: 287-95 CrossRef Medline

13. Constable RT, Anderson AW, Zhong J, et al. Factors influencing contrast in fast spin-echo MR imaging. Magn Reson Imaging 1992; 10:497-511 CrossRef Medline 